(C) The Author(s)

DOI: $10.1142 / \mathrm{S} 1793042118500835$

\title{
Oddness of residually reducible Galois representations
}

\author{
Tobias Berger \\ School of Mathematics and Statistics, University of Sheffield \\ Hicks Building, Hounsfield Road, Sheffield S3 7RH, UK \\ tberger@cantab.net
}

Received 8 March 2017

Accepted 18 September 2017

Published 10 January 2018

\begin{abstract}
We show that suitable congruences between polarized automorphic forms over a CM field always produce elements in the Selmer group for exactly the \pm -Asai (aka tensor induction) representation that is critical in the sense of Deligne. For this, we relate the oddness of the associated polarized Galois representations (in the sense of the BellaïcheChenevier sign being +1 ) to the parity condition for criticality. Under an assumption similar to Vandiver's conjecture this also provides evidence for the Fontaine-Mazur conjecture for residually reducible polarized Galois representations.
\end{abstract}

Keywords: Galois representations; Bloch-Kato conjecture; Fontaine-Mazur conjecture.

Mathematics Subject Classification 2010: 11F80, 11F55

\section{Introduction}

Ribet proved in [26] that, if a prime $p>2$ divides the numerator of the $k$ th Bernoulli number for $k \geq 4$ an even integer, then $p$ divides the order of the part of the class group of $\mathbf{Q}\left(\mu_{p}\right)$ on which $\operatorname{Gal}\left(\mathbf{Q}\left(\mu_{p}\right) / \mathbf{Q}\right)$ acts by the $(1-k)$ th power of the mod $p$ cyclotomic character $\bar{\epsilon}$. The argument (in fact a slight variance of Ribet's proof outlined in [19]) is as follows: One considers the weight $k$ Eisenstein series for $\mathrm{SL}_{2}(\mathbf{Z})$ and proves the existence of a weight $k$ cuspidal eigenform that is congruent to the Eisenstein series. Ribet then shows that one can find a lattice in the irreducible odd $p$-adic Galois representation $\rho_{f}$ associated to $f$ such that its mod $p$ reduction gives a non-split extension $\left(\begin{array}{cc}1 & * \\ 0 & \bar{\epsilon}^{k-1}\end{array}\right)$. Note that this proves one direction of the Bloch-Kato conjecture for the Riemann zeta function (proven in [6. Theorem 6.1(i)]) in so far as it relates the $p$-divisibility of the value $\frac{\zeta(k)}{\pi^{k}}$ for the even integer $k$ to that of the

This is an Open Access article published by World Scientific Publishing Company. It is distributed under the terms of the Creative Commons Attribution 4.0 (CC-BY) License. Further distribution of this work is permitted, provided the original work is properly cited. 
order of the Selmer group for the critical motive $\mathbf{Q}(1-k)$. For odd $k$ this motive is not critical and Vandiver's conjecture predicts, in fact, that the corresponding Selmer group is trivial. We note that $\rho_{f}$ is polarized in the sense that

$$
\rho_{f}^{\vee} \cong \rho_{f} \otimes \operatorname{det}\left(\rho_{f}\right)^{-1} \cong \rho_{f} \otimes \epsilon^{1-k}
$$

and that the characters occurring in the reduction get swapped under this polarization.

We generalize Ribet's strategy using polarized (essentially conjugate dual) Galois representations of CM fields to prove part of the Bloch-Kato conjecture for general Asai (or tensor induction) $L$-functions, our main result in Theorem 4.1 For the Asai $L$-functions induced from CM fields a similar parity condition to that for the Riemann zeta function is required for criticality. We demonstrate in Corollary 4.3 that this parity condition is linked to the oddness of the polarized Galois representation (where the representation is odd if the sign defined in [3] equals +1 , see Definition 2.2).

Before we explain our work we want to discuss a bit more of this parity issue in the situation considered by Ribet. By the Fontaine-Mazur conjecture in [14] all representations $\sigma: G_{\mathbf{Q}} \rightarrow \mathrm{GL}_{2}\left(\overline{\mathbf{Q}}_{p}\right)$ that are "geometric" (i.e. unramified at almost all primes and potentially semi-stable at $p$ ) are modular and therefore odd (in the sense that $\operatorname{det}(\sigma(\tilde{c}))=-1$ for any complex conjugation $\tilde{c}$ ), or they must be a Tate twist of an even representation with finite image. Calegari proved the following remarkable result about the non-existence of even geometric representations:

Theorem $1.1([\mathbf{9}])$. Let $p>7$ be a prime and $\sigma: G_{\mathbf{Q}} \rightarrow \mathrm{GL}_{2}\left(\overline{\mathbf{Q}}_{p}\right)$ a continuous representation such that $\sigma$ is unramified at all but finitely many primes, and at $p$ is potentially semi-stable with distinct Hodge-Tate weights. Further assume that the residual representation $\bar{\sigma}$ is absolutely irreducible and not of dihedral type, and that $\left.\bar{\sigma}\right|_{D_{p}}$ is not a twist of a representation of the form $\left(\begin{array}{ll}\bar{\epsilon} & * \\ 0 & 1\end{array}\right)$. Then $\sigma$ is odd.

In the residually reducible case one can use Ribet's argument to prove the following:

Proposition 1.2. Let $p>2$ and $\sigma: G_{\mathbf{Q}} \rightarrow \mathrm{GL}_{2}\left(\overline{\mathbf{Q}}_{p}\right)$ an irreducible continuous representation such that $\bar{\sigma}^{\mathrm{ss}}=\mathbf{1} \oplus \bar{\epsilon}^{m}$ for $m \in \mathbf{Z}_{>0}$ with $p-1 \nmid m$. Assume that $\sigma$ is unramified outside of $p$, and either ordinary at $p$ or crystalline at $p$ with Hodge-Tate weights in $[0, p-3]$. Then Vandiver's conjecture $\left(p \nmid \# \mathrm{Cl}\left(\mathbf{Q}\left(\mu_{p}\right)^{+}\right)\right)$implies that $\sigma$ is odd.

Proof. By 26] one can find a lattice such that the $\bmod p$ reduction is a non-split extension of the form $\left(\begin{array}{cc}\mathbf{1} & * \\ 0 & \bar{\epsilon}^{m}\end{array}\right)$ that is unramified away from $p$. One can show that this extension splits at $p$. This follows from Fontaine-Laffaille theory if $\sigma$ is "short crystalline" as in the statement, or from ordinarity, which implies that $\left.\sigma\right|_{D_{p}}$ has an unramified quotient. 
This implies (see e.g. [19, p. 275]) that $p \mid \# \mathrm{Cl}\left(\mathbf{Q}\left(\mu_{p}\right)\right)\left(\bar{\epsilon}^{-m}\right)$. By Vandiver's conjecture $m$ has to be odd, which implies that $\operatorname{det}(\sigma)$ is odd.

Remark 1.3. The assumptions on the ramification of $\sigma$ can be weakened, e.g. by allowing $\left.\sigma\right|_{I_{\ell}} \cong\left(\begin{array}{ll}1 & * \\ 0 & 1\end{array}\right)$ for primes $\ell \neq p$ satisfying $\ell^{m-1} \not \equiv 1 \bmod p$. At $p$ crystallinity alone is not enough to be able to deduce that the extension is split at $p$, as the following example (pointed out to us by Kevin Buzzard) shows: Consider $p=3$ and $\sigma=\rho_{\Delta, 3}$ the 3-adic Galois representation associated to the weight 12 level 1 cuspform $\Delta$. Then $\bar{\rho}_{\Delta, 3}^{\text {ss }}=\mathbf{1} \oplus \bar{\epsilon}$, but the class number of $\mathbf{Q}\left(\mu_{3}\right)$ is one.

We will prove in Corollary 5.3 a higher-dimensional generalization of Proposition 1.2 for residually reducible polarized Galois representations of any dimension. This arises as a consequence of the contrapositive of our main result Theorem 4.1 as we will explain in the following:

Given a representation $\rho: G_{K} \rightarrow \mathrm{GL}_{n}(E)$ for $K / K^{+}$a $\mathrm{CM}$ field and $E$ a finite extension of $\mathbf{Q}_{p}$ one can define two extensions of $\rho \otimes \rho^{c}$ to $G_{K^{+}}$, which we denote by $\operatorname{As}^{ \pm}(\rho)$ (for details see Sec. 2.1). Theorem 4.1 concerns the Bloch-Kato conjecture for these representations and generalizes our previous result in [4 for Asai representations of 2-dimensional representations of an imaginary quadratic field to this setting, including extensions of adjoint representations of polarized regular motives as studied in [17]. We explore in this paper the subtle connection between polarizations, signs and criticality that this exposes.

To explain this we need to introduce some more notations: Let $\Psi: G_{K^{+}} \rightarrow E^{*}$ be a Hecke character and $R: G_{K} \rightarrow \mathrm{GL}_{2 n}(E)$ an irreducible $G_{K}$-representation such that $\left.R^{\vee} \cong R^{c} \otimes \Psi\right|_{G_{K}}$ with $\bar{R}^{\mathrm{ss}}=\left.\bar{\rho} \oplus \bar{\rho}^{c \vee} \otimes \Psi^{-1}\right|_{G_{K}}$ (i.e. $R$ is residually reducible with two summands that get swapped under the polarization). Using Shapiro's lemma one can relate the $K^{+}$-Selmer group for $\mathrm{As}^{ \pm \Psi\left(c_{v}\right)}(\bar{\rho}) \otimes \Psi$ to the part of its $K$-Selmer group on which the action by a complex conjugation $c_{v}$ for $v \mid \infty$ is given by $\pm \Psi\left(c_{v}\right)$. In Lemma 4.2 we show that on $H^{1}\left(K, \mathrm{As}^{-\Psi\left(c_{v}\right)}(\bar{\rho}) \otimes \Psi\right)$ the action by $c_{v}$ agrees with an involution $\perp_{c_{v}}$ corresponding to the polarization condition satisfied by $R$. A result of [2] implies that the involution $\perp_{c_{v}}$ acts on the $G_{K}$-extension constructed from $R$ by the sign associated to $\left(R, \Psi, c_{v}\right)$ (introduced in Definition 2.2). Combining these, we get in Theorem 4.1 a construction of an element in the BlochKato Selmer group of $\mathrm{As}^{ \pm}(\rho) \otimes \Psi$, where the sign is given by $-\Psi\left(c_{v}\right) \operatorname{sign}\left(R, \Psi, c_{v}\right)$. In Proposition 3.3, we prove that this $\mathrm{As}^{ \pm}(\rho) \otimes \Psi$ is critical in the sense of Deligne (for characters $\Psi$ where this corresponds to a twist in the left-hand side of the critical strip of $\operatorname{As}(\rho))$ if and only if $\operatorname{sign}\left(R, \Psi, c_{v}\right)=1$. That automorphic Galois representations $R$ are odd in this sense has been proved in 3 for Galois representations associated to regular algebraic cuspidal polarized representations of $\mathrm{GL}_{m}$ over CM fields. We expect it to hold more generally, see the discussion in Remark 2.7]

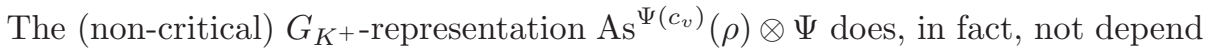
on $\Psi\left(c_{v}\right)$. This allows us in Corollary 5.3 to deduce total oddness of certain residu- 
ally reducible polarized Galois representations if we assume that the Selmer group $H_{\Sigma^{+}}^{1}\left(K^{+}, \operatorname{As}^{\Psi(\tilde{c})}(\bar{\rho}) \otimes \bar{\Psi}\right)$ is trivial for a fixed choice $\tilde{c}$ of complex conjugation.

\section{Notation and Definitions}

If $L$ is a number field then we fix algebraic closures $\bar{L}$ and $\bar{L}_{v}$ for every place $v$ of $L$ and compatible embeddings $\bar{L} \hookrightarrow \bar{L}_{v} \hookrightarrow \mathbf{C}$ and write $D_{L_{v}}$ and $I_{L_{v}}$ (or $I_{v}$ if $L$ is understood) for the corresponding decomposition and inertia subgroups of $G_{L}:=\operatorname{Gal}(\bar{L} / L)$.

We fix a prime $p>2$ and denote by $\epsilon: G_{L} \rightarrow \mathbf{Z}_{p}^{*}$ the $p$-adic cyclotomic character. If $v$ is a finite place of $L$, not dividing $p$, then its value on the arithmetic Frobenius element $\epsilon\left(\right.$ Frob $\left._{v}\right)=q_{v}$, where the latter denotes the size of the residue field of $L$ at $v$. For a $p$-adic representation $V$ of $G_{L}$, we write $V(n):=V \otimes \epsilon^{n}$ for $n \in \mathbf{Z}$. For the coefficients of our $p$-adic representations, we will take $E$ to be a (sufficiently large) finite extension of $\mathbf{Q}_{p}$ inside $\overline{\mathbf{Q}}_{p}$ with ring of integers $\mathcal{O}$ and residue field $\mathbf{F}$. We fix a choice of a uniformizer $\varpi$.

We fix an isomorphism $\overline{\mathbf{Q}}_{p} \cong \mathbf{C}$. If $\chi: \mathbf{A}_{L}^{*} / L^{*} \rightarrow \mathbf{C}^{*}$ is a Hecke character of type $\left(A_{0}\right)$ then we write $\chi^{\text {gal }}$ for the associated Galois character $G_{L} \rightarrow \overline{\mathbf{Q}}_{p}^{*}$ (for details see e.g. [29], but we use the local Artin reciprocity maps mapping uniformizers to arithmetic Frobenius elements).

Let $K / K^{+}$be a CM field, and write $\chi_{K / K^{+}}: G_{K^{+}} \rightarrow\{ \pm 1\}$ for the corresponding quadratic character. We fix a complex conjugation $\tilde{c} \in G_{K^{+}}$. For a $G_{K}$-representation $(\rho, V)$ we define the conjugate representation $\left(\rho^{c}, V^{c}\right)$ by $\rho^{c}(g)=\rho(\tilde{c} g \tilde{c})$.

\subsection{Asai L-function and tensor induction}

We describe the definition of the Asai $L$-function of a cuspidal automorphic representation $\pi$ for $\mathrm{GL}_{m}(K)$ for a CM field $K / K^{+}$(adapting the treatment for $m=2$ in [21], see also [25, Sec. 6]). The Langlands dual of the algebraic group $R_{K / K^{+}} \mathrm{GL}_{m}$ is given by ${ }^{L}\left(R_{K / K^{+}} \mathrm{GL}_{m}\right)=\left(\mathrm{GL}_{m}(\mathbf{C}) \times \mathrm{GL}_{m}(\mathbf{C})\right) \rtimes G_{K^{+}}$. There are $m^{2}$-dimensional representations

$$
r^{ \pm}:{ }^{L}\left(R_{K / K^{+}} \mathrm{GL}_{m} / K\right)(\mathbf{C}) \rightarrow \mathrm{GL}\left(\mathbf{C}^{m} \otimes \mathbf{C}^{m}\right)
$$

given by

$$
r^{ \pm}\left(g, g^{\prime}, \gamma\right)(x \otimes y)=g(x) \otimes g^{\prime}(y) \quad \text { for }\left.\gamma\right|_{K}=1
$$

and

$$
r^{ \pm}(1,1, \gamma)(x \otimes y)= \pm y \otimes x \quad \text { for }\left.\gamma\right|_{K} \neq 1
$$

For each place $v$ we denote by $r_{v}^{ \pm}$the local $L$-group homomorphisms obtained from $r^{ \pm}$by restriction.

Let $\pi$ be a cuspidal automorphic representation for $\operatorname{GL}_{m}\left(\mathbf{A}_{K}\right)$. Then we may consider $\pi$ as a representation of $R_{K / K^{+}} \mathrm{GL}_{m}(\mathbf{A})$ and factorize it as a restricted 
tensor product $\pi=\otimes_{v} \pi_{v}$, where each $\pi_{v}$ is an irreducible admissible representation of $\mathrm{GL}_{m}\left(K \otimes_{K^{+}} K_{v}^{+}\right)$, with corresponding $L$-parameter $\phi_{v}: W_{K_{v}^{+}}^{\prime} \rightarrow\left(\mathrm{GL}_{m}(\mathbf{C}) \times\right.$ $\left.\mathrm{GL}_{m}(\mathbf{C})\right) \rtimes G_{K_{v}^{+}}$. Let $\mathrm{As}^{ \pm}\left(\pi_{v}\right)$ now be the irreducible admissible representation of $\mathrm{GL}_{m^{2}}\left(K_{v}^{+}\right)$corresponding to the parameter $r_{v}^{ \pm} \circ \phi_{v}$ under the local Langlands correspondence and put $\mathrm{As}^{ \pm}(\pi):=\otimes_{v} \mathrm{As}^{ \pm}\left(\pi_{v}\right)$. By Langlands functoriality these should be isobaric automorphic representations of $\mathrm{GL}_{m^{2}}(\mathbf{A})$, the \pm Asai transfers of $\pi$. For $m=2$ this has been proven by Krishnamurty [20] and Ramakrishnan [24].

We will, however, only be referring to the corresponding Langlands $L$-function, which is defined by

$$
L\left(s, \pi, r^{ \pm}\right)=\prod_{v} L\left(s, \pi_{v}, r_{v}^{ \pm}\right)
$$

with $L\left(s, \pi_{v}, r_{v}^{ \pm}\right)=L\left(s, r^{ \pm} \circ \phi_{v}\right)$. For unramified $v$ and $\pi_{v}$ spherical, the latter is given by

$$
\operatorname{det}\left(I-r^{ \pm}\left(A\left(\pi_{v}\right)\right) \mathrm{Nm}(v)^{-s}\right)^{-1}
$$

where $A\left(\pi_{v}\right)$ is the semisimple conjugacy class (Satake parameter) in ${ }^{L}\left(R_{K / K^{+}}\right.$ $\left.\mathrm{GL}_{m}\right)(\mathbf{C})$ (which is represented by $\left(\tilde{A}\left(\pi_{v}\right), \operatorname{Frob}_{v}\right)$ for $\left.\tilde{A}\left(\pi_{v}\right) \in R_{K / K^{+}} \mathrm{GL}_{m}\right)$. For more details and its analytic properties we refer the reader to [16].

We will also consider the twisted Asai $L$-function $L\left(s, \pi, r^{ \pm} \otimes \Psi\right)$ for $\Psi: G_{K^{+}} \rightarrow$ $\overline{\mathbf{Q}}_{p}^{*}$. For unramified places its Euler factors are given by

$$
\operatorname{det}\left(I-\Psi\left(\operatorname{Frob}_{v}\right) r^{ \pm}\left(A\left(\pi_{v}\right)\right) \mathrm{Nm}(v)^{-s}\right)^{-1} .
$$

The operation for Galois representations corresponding to the Asai transfer is usually called tensor (or multiplicative) induction: Consider a representation $\rho$ : $G_{K} \rightarrow \mathrm{GL}(V)$ for an $n$-dimensional vector space $V$ over a field. Then we define the following extensions of $V \otimes V^{c}$ to $G_{K^{+}}$:

$$
\operatorname{As}^{ \pm}(\rho): G_{K^{+}} \rightarrow \mathrm{GL}\left(V \otimes V^{c}\right)
$$

given by

$$
\mathrm{As}^{ \pm}(\rho)(g)(x \otimes y)=\rho(g) x \otimes \rho^{c}(g)(y) \quad \text { for }\left.g\right|_{K}=1
$$

and

$$
\operatorname{As}^{ \pm}(\rho)(\tilde{c})(x \otimes y)= \pm y \otimes x .
$$

We note that $\operatorname{As}^{-}(\rho) \cong \operatorname{As}^{+}(\rho) \otimes \chi_{K / K^{+}}$.

\subsection{Bloch-Kato Selmer groups}

Let $L$ be either $K$ or $K^{+}$. Consider a continuous finite-dimensional representation $V$ of $G_{L}$ over $E$. Let $T \subseteq V$ be a $G_{L}$-stable $\mathcal{O}$-lattice and put $W=V / T$ and 
$W_{n}=\left\{x \in W: \varpi^{n} x=0\right\}$. Let $\Sigma$ be a finite set of places of $L$. Following BlochKato (see also 12, Sec. 2.1) we define for $M=W, W_{n}$ the following Selmer groups:

$$
H_{\Sigma}^{1}(L, M)=\operatorname{ker}\left(H^{1}(L, M) \rightarrow \prod_{v \notin \Sigma} H^{1}\left(L_{v}, M\right) / H_{f}^{1}\left(L_{v}, M\right)\right),
$$

where $H_{f}^{1}\left(L_{v}, W\right):=\operatorname{im}\left(H_{f}^{1}\left(L_{v}, V\right) \rightarrow H^{1}\left(L_{v}, W\right)\right)$ and

$$
H_{f}^{1}\left(L_{v}, V\right)= \begin{cases}\operatorname{ker}\left(H^{1}\left(L_{v}, V\right) \rightarrow H^{1}\left(I_{v}, V\right)\right) & \text { if } v \nmid p, \infty, \\ \operatorname{ker}\left(H^{1}\left(L_{v}, V\right) \rightarrow H^{1}\left(L_{v}, V \otimes B_{\text {cris }}\right)\right) & \text { if } v \mid p, \\ 0 & \text { if } v \mid \infty .\end{cases}
$$

When $V$ is short crystalline (i.e. restrictions to $D_{v}$ for $v \mid p$ described by FontaineLaffaille theory as in 12, Sec. 1.1.2], but with the more restrictive filtration condition

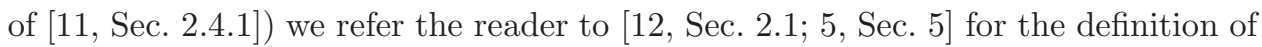
the local subgroups $H_{f}^{1}\left(L_{v}, W_{n}\right)$ for finite modules using Fontaine-Laffaille theory.

\subsection{Polarized Galois representations and signs}

Definition 2.1. Let $\Psi: G_{K^{+}} \rightarrow E^{*}$ be a character and $R: G_{K} \rightarrow \mathrm{GL}_{m}(E)$ an absolutely irreducible representation. We call $(R, \Psi)$ polarized if

$$
\left.R^{\vee} \cong R^{c} \otimes \Psi\right|_{G_{K}} .
$$

In this section we consider complex conjugations $c_{v} \in G_{K^{+}}$for $v \mid \infty$ and conjugate representations $R^{c_{v}}$ defined by $R^{c_{v}}(g)=R\left(c_{v} g c_{v}\right)$ (which are all isomorphic to $R^{c}$ defined using our fixed complex conjugation $\tilde{c}$ ). By Schur's Lemma there exists a matrix $A_{v} \in \mathrm{GL}_{m}\left(\overline{\mathbf{Q}}_{p}\right)$, unique up to scalar, such that $R^{\vee}=\left.A_{v} R^{c_{v}} A_{v}^{-1} \Psi\right|_{G_{K}}$, depending on $v \mid \infty$. This implies that

$$
R^{c_{v}}=\left.\left(A_{v}^{T}\right)^{-1} R^{\vee} A_{v}^{T} \Psi^{-1}\right|_{G_{K}},
$$

using $\left(\left.\Psi\right|_{G_{K}}\right)^{c_{v}}=\left.\Psi\right|_{G_{K}}$. One can then apply Schur's Lemma again (see e.g. [8]) to deduce that the matrix $A_{v}$ is in fact symmetric or antisymmetric.

Definition 2.2. Let $(R, \Psi)$ be polarized. If the matrix $A_{v}$ described above is symmetric we call $(R, \Psi)$ odd at $v$ (or $R$ odd at $v$ with respect to $\Psi$ ) and define $\operatorname{sign}\left(R, \Psi, c_{v}\right)=1$ (otherwise we put $\operatorname{sign}\left(R, \Psi, c_{v}\right)=-1$ ). If $(R, \Psi)$ is odd for all $v \mid \infty$ we call $(R, \Psi)$ totally odd.

Lemma 2.3. The signs $\operatorname{sign}\left(R, \Psi, c_{v}\right)$ are independent of $v \mid \infty$ if and only if the values $\Psi\left(c_{v}\right)$ are.

Proof. For $R^{\tilde{c}^{\prime}}$ the matrix $A_{\tilde{c}^{\prime}}$ equals $A_{\tilde{c}} R\left(\tilde{c}^{\prime} \tilde{c}\right)$. Since

$$
\begin{aligned}
\left(A_{\tilde{c}} R\left(\tilde{c}^{\prime} \tilde{c}\right)\right)^{T} & =R\left(\tilde{c}^{\prime} \tilde{c}\right)^{T} A_{\tilde{c}}^{T}=R^{\vee}\left(\tilde{c} \tilde{c}^{\prime}\right) A_{\tilde{c}}^{T}=A_{\tilde{c}} R\left(\tilde{c}^{\prime} \tilde{c}\right) A_{\tilde{c}}^{-1} A_{\tilde{c}}^{T} \Psi\left(\tilde{c} \tilde{c}^{\prime}\right) \\
& =A_{\tilde{c}} R\left(\tilde{c}^{\prime} \tilde{c}\right) \cdot \operatorname{sign}(R, \Psi, \tilde{c}) \Psi\left(\tilde{c} \tilde{c}^{\prime}\right)
\end{aligned}
$$


we deduce that

$$
\operatorname{sign}\left(R, \Psi, \tilde{c}^{\prime}\right)=\operatorname{sign}(R, \Psi, \tilde{c}) \Psi\left(\tilde{c} \tilde{c}^{\prime}\right) .
$$

Remark 2.4. This lemma shows that our notion of "totally odd polarized" agrees with that in 1, Sec. 2], except that we do not demand that $\Psi\left(c_{v}\right)=-1$ for all $v \mid \infty$, which can, however, easily be achieved by replacing $\Psi$ by $\Psi_{K_{K / K^{+}}}$.

Example 2.5 (Essentially self-dual $\boldsymbol{G}_{K^{+}}$-representation). (1) Let $\tilde{R}$ : $G_{K^{+}} \rightarrow \mathrm{GL}_{m}(E)$ absolutely irreducible such that

$$
\tilde{R}^{\vee} \cong \tilde{R} \otimes \Psi
$$

This implies that $\tilde{R}$ is either (generalized) symplectic or orthogonal, i.e. that there exists a sympletic or orthogonal pairing for the vector space underlying $\tilde{R}$. Lemma 2.6 in 8 shows that $\left(\left.\tilde{R}\right|_{G_{K}}, \Psi\right)$ is odd at $v$ either if $\tilde{R}$ is symplectic and $\Psi\left(c_{v}\right)=-1$ or if $\tilde{R}$ is orthogonal and $\Psi\left(c_{v}\right)=1$.

In particular, this shows that the notion of oddness in Definition 2.2 generalizes the notion of oddness for 2-dimensional representations $\rho: G_{K^{+}} \rightarrow$ $\mathrm{GL}_{2}\left(\overline{\mathbf{Q}}_{p}\right)$ requiring $\operatorname{det}(\rho)(\tilde{c})=-1$.

(2) Consider a 2-dimensional irreducible representation $R: G_{K} \rightarrow \mathrm{GL}_{2}\left(\overline{\mathbf{Q}}_{p}\right)$ such that $R^{\vee} \cong R^{c} \otimes \operatorname{det}(R)^{-1}$ and $\operatorname{det} R$ extends to $G_{K^{+}}$. Then $R^{c} \cong R$, so $R$ extends to $G_{K^{+}}$. Let $\tilde{R}: G_{K^{+}} \rightarrow \mathrm{GL}_{2}\left(\overline{\mathbf{Q}}_{p}\right)$ be either of the two extensions. This satisfies $\tilde{R}^{\vee} \cong \tilde{R} \otimes \operatorname{det}(\tilde{R})^{-1}$ (but not $\tilde{R}^{\vee} \cong \tilde{R} \otimes \operatorname{det}(\tilde{R})^{-1} \chi_{K / K^{+}}$since $\left.\tilde{R} ¥ \tilde{R} \otimes \chi_{K / K^{+}}\right)$. This means that $\operatorname{sign}(R)=1$ is again equivalent to the oddness of the polarization character $\operatorname{det}(\tilde{R})$.

We recall from e.g. [29], Theorem 2.2] that for $(\pi, \chi)$ a RAECSDC automorphic representation of $\mathrm{GL}_{m}\left(\mathbf{A}_{K}\right)$ there exists a continuous semisimple representation

$$
\rho_{\pi}: G_{K} \rightarrow \mathrm{GL}_{m}\left(\overline{\mathbf{Q}}_{p}\right)
$$

such that

(1) $\left.\rho_{\pi}^{\vee} \cong \rho_{\pi}^{c} \otimes \epsilon^{1-m} \chi^{\text {gal }}\right|_{G_{K}}$.

(2) $\left.\rho_{\pi}\right|_{D_{v}}$ is de Rham for each $v \mid p$.

(3) For each finite place $v$ of $K$, we have

$$
\mathrm{WD}\left(\left.\rho_{\pi}\right|_{G_{K_{v}}}\right)^{\mathrm{F}-\mathrm{ss}} \cong \operatorname{rec}_{K_{v}}^{\vee}\left(\pi_{v} \otimes|\operatorname{det}|^{(1-m) / 2}\right),
$$

where $\operatorname{rec}_{K_{v}}^{\vee}$ is the local Langlands correspondence for $\mathrm{GL}_{m}\left(K_{v}\right)$ using the arithmetic Frobenius normalization.

Theorem 2.6 ([3; 1, Theorem 2.1.1(1)]). Let $(\pi, \chi)$ be a RAECSDC representation of $\mathrm{GL}_{m}\left(\mathbf{A}_{K}\right)$. Then (any irreducible factor of) $\rho_{\pi}$ is totally odd with respect to $\epsilon^{1-m} \chi^{\text {gal }}$.

Remark 2.7. In certain cases, Galois representations have been constructed for irregular $\pi$ (see, in particular, the recent papers of [15, 23] in the case that the 
descent of $\pi$ to a unitary group has a non-degenerate limit of discrete series as archimedean component).

In Theorem 4.1, we consider representations that are residually reducible and not necessarily Hodge-Tate regular (for an example see [4, Theorem 5.1]). We are therefore interested in the extension of Bellaïche-Chenevier's result to $\pi$ that are not regular.

If $p>2$ and $\rho_{\pi}$ is residually irreducible then the signs of $\rho_{\pi}$ and its reduction agree (see e.g. 3. Sec.2.3). This implies that in such cases oddness is also known for irregular $\pi$ if there is a congruence to a RAECSDC representation. Since oddness of $\rho_{\pi}$ corresponds to the associated $G_{K^{+}}$-representation being valued in the $L$-group of (an appropriate covering group of) the unitary group (see [7, Sec. 8.3]) the work of Lafforgue on pseudorepresentations ([22, Proposition 11.7]) should more generally allow to relax the regularity assumption in Theorem 2.6 .

\section{Bloch-Kato Conjecture}

Let $\pi$ be a regular $L$-algebraic cuspidal automorphic representation of $\mathrm{GL}_{m}\left(\mathbf{A}_{K}\right)$. Harris et al. [18] and Scholze [28] have constructed Galois representations $\rho_{\pi}$ : $G_{K} \rightarrow \mathrm{GL}_{m}(E)$ associated to $\pi$ (for $E$ a sufficiently large finite extension of $\mathbf{Q}_{p}$ ) such that its Frobenius eigenvalues match the Hecke eigenvalues of $\pi$ (we again take the arithmetic Frobenius normalization).

In this section, we state the Bloch-Kato conjecture (following the exposition in 13]) for the Asai $L$-value $L\left(1, \pi, r^{ \pm} \otimes \Psi\right)$ for $\Psi: G_{K^{+}} \rightarrow E^{*}$ a continuous Galois character and analyze the criticality of $\mathrm{As}^{ \pm}\left(\rho_{\pi}\right)(-m)$.

Let $F$ be a number field (including the field of definition of the Satake parameters of $\pi$ ), and $\mathfrak{p} \mid p$ a place of $F$ with $F_{\mathfrak{p}} \cong E$. Let $\operatorname{As}^{ \pm}\left(\rho_{\pi}\right): G_{K^{+}} \rightarrow \operatorname{GL}_{m}(E)$ be the Asai plus/minus representation defined in Sec. 2.1. By [10, Conjecture 4.5], (applied to the conjectural Asai transfer $\mathrm{As}^{ \pm}(\pi)$ to $\mathrm{GL}_{m^{2}}(\mathbf{A})$ ) there should exists a motive $\mathcal{M}\left(\pi, r^{ \pm} \otimes \Psi\right)$ of rank $m^{2}$ with coefficients in $F$ such that if $\rho_{\mathcal{M}\left(\pi, r^{ \pm} \otimes \Psi\right), \mathfrak{p}}$ : $G_{K^{+}} \rightarrow \mathrm{GL}_{m^{2}}(E)$ is its $\mathfrak{p}$-adic realization then $\rho_{\mathcal{M}\left(\pi, r^{ \pm} \otimes \Psi\right), \mathfrak{p}}\left(\mathrm{Frob}_{v}^{-1}\right)$ is conjugate in $\mathrm{GL}_{m^{2}}(E)$ to $\left(r^{ \pm} \otimes \Psi\right)\left(A\left(\pi_{v}\right)\right)$, where $A\left(\pi_{v}\right)$ is the Satake parameter in ${ }^{L}\left(R_{K / K^{+}} \mathrm{GL}_{m}\right)(\mathbf{C})$. Note that this $\mathfrak{p}$-adic realization is dual to $\operatorname{As}^{ \pm}\left(\rho_{\pi}\right) \otimes \Psi$ due to the arithmetic Frobenius normalization we chose for $\rho_{\pi}$.

Put $\mathcal{M}:=\mathcal{M}\left(\pi, r^{ \pm} \otimes \Psi\right)$. Let $H_{\mathrm{B}}(\mathcal{M})$ and $H_{\mathrm{dR}}(\mathcal{M})$ be the Betti and de Rham realizations, and let $H_{\mathrm{B}}(\mathcal{M})^{ \pm}$be the eigenspaces for the complex conjugation $\tilde{c}$. Following Deligne we call $\mathcal{M}$ critical if $\operatorname{dim}\left(H_{\mathrm{B}}(\mathcal{M})^{+}\right)=\operatorname{dim}\left(H_{\mathrm{dR}}(\mathcal{M}) / \mathrm{Fil}^{0}\right)$. Assume that this is the case for $\mathcal{M}(1)$ (which requires, in particular, a choice of $r^{+}$or $r^{-}$, see Proposition 3.3).

Assume $p \gg 0$ (see 13, Sec. 4 for details, but in particular, so that $\left(\operatorname{As}^{ \pm}\left(\rho_{\pi}\right) \otimes\right.$ $\Psi)\left.\right|_{D_{v}}$ for $v \mid p$ can be described using Fontaine-Laffaille theory) and $\pi$ is not ramified at any places dividing $p$. Let $\mathcal{O}_{(\mathfrak{p})}$ be the localization at $\mathfrak{p}$ of the ring

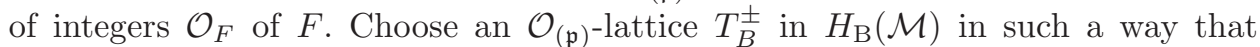

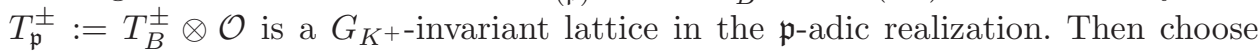


an $\mathcal{O}_{(\mathfrak{p})}$-lattice $T_{\mathrm{dR}}^{ \pm}$in $H_{\mathrm{dR}}(\mathcal{M})$ in such a way that $\mathbb{V}\left(T_{\mathrm{dR}}^{ \pm} \otimes \mathcal{O}\right)=T_{\mathfrak{p}}^{ \pm}$as $G_{K_{v}^{+-}}$ representations for $v \mid p$, where $\mathbb{V}$ is the version of the Fontaine-Laffaille functor used in 12 .

Let $\Omega$ be a Deligne period scaled according to the above choice, i.e. the determinant of the isomorphism

$$
H_{\mathrm{B}}(\mathcal{M}(1))^{+} \otimes \mathbf{C} \cong\left(H_{\mathrm{dR}}(\mathcal{M}(1)) / \mathrm{Fil}^{0}\right) \otimes \mathbf{C},
$$

calculated with respect to the bases of $\left(T_{B}^{ \pm}\right)^{+}$and $T_{\mathrm{dR}}^{ \pm} / \mathrm{Fil}^{1}$, so well-defined up to $\mathcal{O}_{(\mathfrak{p})}^{*}$.

Conjecture 3.1 ([6; $; 3$, Conjecture 4.1]). If $\Sigma^{+}$is a finite set of primes, containing all $v$ where $\pi_{v}$ or $K / K^{+}$is ramified, but not containing $p$ then

$$
\operatorname{ord}_{\mathfrak{p}}\left(\frac{L^{\Sigma^{+}}\left(1, \pi, r^{ \pm} \otimes \Psi\right)}{\Omega}\right)=\operatorname{ord}_{\mathfrak{p}}\left(\frac{\# H_{\Sigma^{+}}^{1}\left(K^{+}, T_{\mathfrak{p}}^{*} \otimes(E / \mathcal{O})\right)}{\# H^{0}\left(K^{+}, T_{\mathfrak{p}}^{*} \otimes(E / \mathcal{O})\right)}\right),
$$

where $T_{\mathfrak{p}}^{*}=\operatorname{Hom}_{\mathcal{O}}\left(T_{\mathfrak{p}}, \mathcal{O}\right)$, with the dual action of $G_{K^{+}}$, and \# denotes a Fitting ideal.

Remark 3.2. Note that the Langlands $L$-function $L\left(1, \pi, r^{ \pm} \otimes \Psi\right)$ equals $L(1, \mathcal{M})$ and relates to the Galois modules on the right-hand side given by $\mathcal{M}^{\vee} \cong$ $\operatorname{As}^{ \pm}\left(\rho_{\pi}\right) \otimes \Psi$. We will write the Selmer group in the numerator of the right-hand side as $H_{\Sigma^{+}}^{1}\left(K^{+}, \mathrm{As}^{ \pm}\left(\rho_{\pi}\right) \otimes \Psi \otimes(E / \mathcal{O})\right)$ in the following.

(2) If $m=2$ (so that $\rho_{\pi}^{\vee} \cong \rho_{\pi} \otimes \operatorname{det}\left(\rho_{\pi}\right)^{-1}$ ) and the central character of $\pi$ extends to $G_{K^{+}}$(the situation in [4]) or if $(\pi, \chi)$ is an RAECSDC representation and $\Psi=\epsilon^{1-m} \chi^{\text {gal }}$ then $\operatorname{As}\left(\rho_{\pi}\right) \otimes \Psi$ is self-dual (using properties of the twisted tensor induction and that the transfer of $\left.\Psi\right|_{G_{K}}$ to $G_{K^{+}}$is $\Psi^{2}$, see [4] Lemma $4.1])$.

For the case of $\left.\Psi\right|_{G_{K}}=\epsilon^{-m}$ we analyze the criticality of the Asai motive $\mathcal{M}\left(\pi, r^{ \pm} \otimes \Psi\right)(1)=\mathcal{M}\left(\pi, r^{ \pm}\right)(m+1)$ (or equivalently $\left.\operatorname{As}^{ \pm}\left(\rho_{\pi}\right)(-m)\right)$ :

Proposition 3.3. Let $p>2$ and $\rho=\rho_{\pi}: G_{K} \rightarrow \mathrm{GL}_{n}(E)$ be a residually irreducible continuous representation arising from a regular motive with coefficients in $F$ that is pure of some weight. We assume that the Hodge decomposition of $H_{B}\left(\mathcal{M}\left(\pi, r^{ \pm}\right)\right)$ involves $H^{a, b}$ with $0 \leq a, b \leq 2 \mathrm{wt}(\rho)$. Then for an integer $m$ in the right-hand side of the critical strip for $H_{B}\left(\mathcal{M}\left(\pi, r^{ \pm}\right)(1)\right)$, i.e. $m \geq \operatorname{wt}(\rho)$ and

$$
m \leq m_{0}:=\min \left\{a \mid H^{a, b} \neq 0, a>\operatorname{wt}(\rho)\right\}-1,
$$

the motive $\mathrm{As}^{(-1)^{m+1}}(\rho)(-m)$ is critical in the sense of Deligne, whereas $\operatorname{As}^{(-1)^{m}}(\rho)(-m)$ is not (so exactly the odd (respectively even) integers in $\left[\mathrm{wt}(\rho), m_{0}\right]$ are critical for $\mathrm{As}^{+}(\rho)(-m)$ (respectively, $\left.\left.\mathrm{As}^{-}(\rho)(-m)\right)\right)$.

Proof. We need to show

$$
\operatorname{dim} H_{B}\left(\mathcal{M}^{\vee}\left(\pi, r^{(-1)^{m+1}}\right)(-m)\right)^{+}=\operatorname{dim} H_{\mathrm{dR}}\left(\mathcal{M}^{\vee}\left(\pi, r^{(-1)^{m+1}}\right)(-m)\right) / \mathrm{Fil}^{0} .
$$


(In the following, we will write $r$ for $r^{(-1)^{m+1}}$.) We note that the center of the Hodge decomposition of $H_{\mathrm{dR}}\left(\mathcal{M}^{\vee}(\pi, r)(-m)\right)$ has dimension $h^{\mathrm{wt}(\rho) \text {, wt }(\rho)}=n$ since the motive corresponding to $\rho$ is assumed to be pure and regular. If we list $(a, b)$ occurring in the Hodge decomposition of $H_{\mathrm{dR}}\left(\mathcal{M}^{\vee}(\pi, r)(-m)\right)$ in order of increasing $a$ then under our assumption on $m$ the pair immediately to the left of center has a negative $a$. This implies that

$$
\begin{aligned}
\operatorname{dim} H_{\mathrm{dR}}\left(\mathcal{M}^{\vee}(\pi, r)(-m)\right) / \mathrm{Fil}^{0} & =\frac{1}{2}\left(\operatorname{dim} H_{\mathrm{dR}}\left(\mathcal{M}^{\vee}(\pi, r)(-m)\right)-h^{\mathrm{wt}(\rho), \mathrm{wt}(\rho)}\right) \\
& =\frac{n(n-1)}{2} .
\end{aligned}
$$

To calculate $\operatorname{dim} H_{B}\left(\mathcal{M}^{\vee}(\pi, r)(-m)\right)^{+}$we follow the argument in [17 Sec. 1.3]: Write $M_{B}:=H_{B}\left(\mathcal{M}(\rho)^{\vee}\right)$ for the Betti realization of the motive corresponding to $\rho$, which is a module over the number field $F$. Choose an $F$-basis $e_{1}, \ldots, e_{n}$ of $M_{B}$. Let $t_{B}=2 \pi i t$ be a rational basis for $K^{+}(1)_{B}=(2 \pi i) K^{+}$and $e_{\chi}$ a basis vector for the Betti realization of the Artin motive $K^{+}\left(\chi_{K / K^{+}}\right)$of rank 1 over $K^{+}$attached to the character $\chi_{K / K^{+}}$. As a model of $H_{B}\left(\mathcal{M}^{\vee}(\pi, r)(-m)\right)$ we take

$$
H_{B}\left(\mathcal{M}^{\vee}(\pi, r)(-m)\right)=M_{B} \otimes M_{B}^{c}(-m) \otimes K^{+}\left(\chi_{K / K^{+}}\right)_{B}^{\otimes m+1}
$$

with the action

$$
\begin{aligned}
\tilde{c}\left(e_{a} \otimes e_{b}^{c} \otimes t_{B}^{-m} \otimes e_{\chi}^{m+1}\right) & =e_{b} \otimes e_{a}^{c} \otimes(-1)^{m} t_{B}^{-m} \otimes(-1)^{m+1} e_{\chi}^{m+1} \\
& =-e_{b} \otimes e_{a}^{c} \otimes t_{B}^{-m} \otimes e_{\chi}^{m+1} .
\end{aligned}
$$

This implies that the vectors

$$
\left\{e_{a b}^{+}=\left[e_{a} \otimes e_{b}^{c}-e_{b} \otimes e_{a}^{c}\right] \otimes t_{B}^{-m} \otimes e_{\chi}^{m+1}, a<b\right\}
$$

form a basis for $H_{B}\left(\mathcal{M}^{\vee}\left(\pi, r^{(-1)^{m+1}}\right)(-m)\right)^{+}$, so that its dimension is therefore also $\frac{n(n-1)}{2}$ (whereas $H_{B}\left(\mathcal{M}^{\vee}\left(\pi, r^{(-1)^{m}}\right)(-m)\right)^{+}$has dimension $\frac{n(n+1)}{2}$ with basis

$$
\left.\left\{f_{a b}^{+}=\left[e_{a} \otimes e_{b}^{c}+e_{b} \otimes e_{a}^{c}\right] \otimes t_{B}^{-m} \otimes e_{\chi}^{m}, a \leq b\right\}\right) .
$$

\section{Construction of Elements in Critical Selmer Groups for General Asai Representations}

This section proves the main result (Theorem 4.1) which establishes the Galois part of the proof of one direction of the Bloch-Kato conjecture for general Asai representations (i.e. we assume the existence of a residually reducible representation $R$ that could be constructed by establishing suitable congruences of polarized automorphic forms). Note that the assumption in the theorem on the reduction of $R$ is that $\bar{R}^{\mathrm{ss}}$ has two summands that get swapped under the involution $\sigma \mapsto \sigma^{c \vee} \otimes \Psi^{-1}$ corresponding to the polarization of $(R, \Psi)$.

Theorem 4.1. Let $p>2, \Psi: G_{K^{+}} \rightarrow E^{*}$ a character, and $\rho: G_{K} \rightarrow \operatorname{GL}_{n}(E) a$ residually irreducible continuous representation with

$$
\bar{\rho} \not \equiv \bar{\rho}^{c \vee} \Psi^{-1} \bmod \varpi \text {. }
$$


Assume there exists an absolutely irreducible representation

$$
R: G_{K} \rightarrow \mathrm{GL}_{2 n}(E)
$$

that is unramified away from a finite set of places $\Sigma$ and short crystalline at $v \mid p$ such that

$$
\left.R^{\vee} \cong R^{c} \otimes \Psi\right|_{G_{K}}
$$

and

$$
\left.\bar{R}^{\mathrm{ss}} \cong \bar{\rho} \oplus \bar{\rho}^{c \vee} \otimes \bar{\Psi}^{-1}\right|_{G_{K}} .
$$

If $\Sigma^{+}$is the set of places of $K^{+}$lying below $\Sigma$ then

$$
p \mid \# H_{\Sigma^{+}}^{1}\left(K^{+}, \mathrm{As}^{-\Psi(\tilde{c}) \operatorname{sign}(R, \Psi, \tilde{c})}(\rho) \otimes \Psi \otimes(E / \mathcal{O})\right) .
$$

Proof. We generalize the proof of [4, Theorem 6.1].

By assumption $\left.\bar{R}^{\text {ss }} \equiv \bar{\rho} \oplus \bar{\rho}^{\tilde{c} \vee} \otimes \bar{\Psi}^{-1}\right|_{G_{K}}$. By Ribet's lemma (see e.g. 30, Theorem 1.1]) we know there exists a lattice for $R$ such that

$$
R \equiv\left(\begin{array}{cc}
\bar{\rho} & * \\
0 & \left.\bar{\rho}^{\tilde{c} \vee} \bar{\Psi}^{-1}\right|_{G_{K}}
\end{array}\right) \quad \bmod \varpi
$$

and this extension is not split.

We claim now that this gives rise to an element in

$$
H_{\Sigma}^{1}\left(K, \mathrm{As}^{+}(\rho) \otimes \Psi \otimes(E / \mathcal{O})\right)^{-\Psi(\tilde{c}) \operatorname{sign}(R, \Psi, \tilde{c})},
$$

which is isomorphic to $H_{\Sigma^{+}}^{1}\left(K^{+}, \mathrm{As}^{-\Psi(\tilde{c}) \operatorname{sign}(R, \Psi, \tilde{c})}(\rho) \otimes \Psi \otimes(E / \mathcal{O})\right)$ by Shapiro's lemma (see [4 Lemma 3.1]).

First note that (4.1) gives a non-trivial class in $H^{1}\left(K, \operatorname{Hom}_{\mathbf{F}}\left(\left.\bar{\rho}^{\tilde{c} \vee} \bar{\Psi}^{-1}\right|_{G_{K}}, \bar{\rho}\right)\right)$. By the assumptions on the ramification and crystallinity of $\tilde{R}$ we have, in fact, a class in $H_{\Sigma}^{1}\left(K, \operatorname{Hom}_{\mathbf{F}}\left(\bar{\rho}^{\tilde{c} \vee} \bar{\Psi}^{-1}, \bar{\rho}\right)\right)$. Since

$$
\left.\operatorname{Hom}_{\mathbf{F}}\left(\bar{\rho}^{\tilde{c} \vee} \bar{\Psi}^{-1}, \bar{\rho}\right) \cong \bar{\rho} \otimes \bar{\rho}^{\tilde{c}} \otimes \bar{\Psi} \cong\left(\operatorname{As}^{+}(\bar{\rho}) \otimes \bar{\Psi}\right)\right|_{G_{K}}
$$

we obtain an element in

$$
\left.H_{\Sigma}^{1}\left(K, \operatorname{As}^{+}(\bar{\rho}) \otimes \bar{\Psi}\right) \cong H_{\Sigma}^{1}\left(K, \operatorname{As}^{+}(\rho) \otimes \Psi\right) \otimes(E / \mathcal{O})[\varpi]\right),
$$

which injects into $H_{\Sigma}^{1}\left(K, \mathrm{As}^{+}(\rho) \otimes \Psi \otimes(E / \mathcal{O})\right)$ since $H^{0}\left(K^{+}, \mathrm{As}^{+}(\bar{\rho}) \otimes \bar{\Psi}\right)=0$. To see the latter, assume that $\operatorname{Hom}_{G_{K^{+}}}\left(\mathbf{1}, \mathrm{As}^{+}(\bar{\rho}) \otimes \bar{\Psi}\right) \neq 0$. This implies $\operatorname{Hom}_{G_{K}}(\mathbf{1}, \bar{\rho} \otimes$ $\left.\bar{\rho}^{c} \otimes \bar{\Psi}\right) \neq 0$, contradicting our assumption that $\bar{\rho}$ and $\bar{\rho}^{c \vee} \bar{\Psi}^{-1}$ are irreducible and non-isomorphic.

Lemma 4.2. On $H^{1}\left(K, \mathrm{As}^{+}(\bar{\rho}) \otimes \bar{\Psi}\right)$ the $\tilde{c}$-action coincides with $-\Psi(\tilde{c})$ times the "polarization involution" $\perp_{\tilde{c}}$ arising from the involution on $\mathbf{F}\left[G_{K}\right]$ given by $g \mapsto$ $\tau(g):=\tilde{c} g^{-1} \tilde{c}^{-1} \bar{\Psi}^{-1}(g)$ for $g \in G_{K}$. 
Proof. We recall from [2, Sec. 1.8] how $\tau$ induces an involution on $H^{1}(K, \operatorname{As}(\bar{\rho}) \otimes \bar{\Psi})$. (For this action we only require the $G_{K}$-module, so we will write the coefficients as $\operatorname{Hom}_{\mathbf{F}}\left(\bar{\rho}^{\tilde{c} \vee} \bar{\Psi}^{-1}, \bar{\rho}\right)$.) In our case, $\tau$ is an anti-involution of algebras, and the corresponding involution on representations $\sigma: G_{K} \rightarrow \mathrm{GL}_{m}(\mathbf{F})$ is given by $\sigma \mapsto \sigma^{\perp}:=$ $(\sigma \circ \tau)^{T}$.

Equation (26) on p. 51 of [2] explains that the induced involution on $H^{1}\left(K, \operatorname{Hom}_{\mathbf{F}}\left(\bar{\rho}^{\tilde{c} \vee} \bar{\Psi}^{-1}, \bar{\rho}\right)\right)$ can be described as follows: Associate to a cocycle $\phi \in$ $Z^{1}\left(G_{K}, \operatorname{Hom}_{\mathbf{F}}\left(\bar{\rho}^{\tilde{c} \vee} \bar{\Psi}^{-1}, \bar{\rho}\right)\right)$ the representation

$$
\rho_{\phi}: G_{K} \rightarrow \mathrm{GL}_{2 n}(\mathbf{F}): g \mapsto\left(\begin{array}{cc}
\bar{\rho}(g) & b(g) \\
0 & \bar{\rho}^{\tilde{c} \vee} \bar{\Psi}^{-1}(g)
\end{array}\right)
$$

with $b(g)=\phi(g) \bar{\rho}^{\tilde{c} \vee} \bar{\Psi}^{-1}(g)$. Then $\phi^{\perp}:=\phi^{\perp \tilde{c}}$ given by

$$
\phi^{\perp}(g):=b^{T}\left(\tilde{c} g^{-1} \tilde{c}^{-1}\right) \bar{\rho}^{\tilde{c} \vee}\left(g^{-1}\right)
$$

defines an involution $\perp$ on $\left.H_{\Sigma}^{1}\left(K, \operatorname{Hom}_{\mathbf{F}}\left(\bar{\rho}^{\tilde{c} \vee} \bar{\Psi}^{-1}, \bar{\rho}\right)\right)\right)$.

As in the proof of [4, Lemma 5.2] we calculate that

$$
\phi^{\perp}(g)=\bar{\rho}(g) \phi^{T}\left(\tilde{c} g^{-1} \tilde{c}^{-1}\right) \rho^{\tilde{c} \vee}\left(g^{-1}\right) \bar{\Psi}(g)=-\phi^{T}\left(\tilde{c} g \tilde{c}^{-1}\right),
$$

where we used the cocycle relation for the last equality.

We compare this to the action of $\tilde{c} \in G_{K^{+}}$on $[\phi] \in H_{\Sigma}^{1}\left(K, \mathrm{As}^{+}(\bar{\rho}) \otimes \bar{\Psi}\right)$ given by

$$
(\tilde{c} . \phi)(g)=\left(\mathrm{As}^{+}(\bar{\rho}) \otimes \Psi\right)(\tilde{c}) \phi\left(\tilde{c} g \tilde{c}^{-1}\right) .
$$

Since $\mathrm{As}^{+}(\rho)(\tilde{c})$ acts as transpose on the upper right shoulder,

$$
\phi^{T} \in Z^{1}\left(G_{K}, \operatorname{Hom}_{\mathbf{F}}\left(\bar{\rho}^{\vee},\left(\bar{\rho}^{\tilde{c} \vee} \bar{\Psi}^{-1}\right)^{\vee}\right)=Z^{1}\left(G_{K},\left.\operatorname{As}^{+}(\bar{\rho}) \otimes \bar{\Psi}\right|_{G_{K}}\right),\right.
$$

complex conjugation acts by $\phi \mapsto\left(g \mapsto \Psi(\tilde{c}) \phi^{T}\left(c g c^{-1}\right)\right)$ on a cocyle representing a class $[\phi] \in H_{\Sigma}^{1}\left(K, \operatorname{As}^{+}(\bar{\rho}) \otimes \bar{\Psi}\right)$.

By using a result of Bellaïche and Chenevier we can now show that the extension (4.1) constructed using [30, Theorem 1.1] lies in $H^{1}\left(K, \mathrm{As}^{+}(\rho) \otimes \Psi \otimes\right.$ $(E / \mathcal{O}))^{-\Psi(\tilde{c}) \operatorname{sign}(R, \Psi, \tilde{c})}$. In fact, Urban's Theorem 1.1 constructs an element $c_{G}$ of $\operatorname{Ext}_{\mathbf{F}\left[G_{K}\right]}^{1}\left(\bar{\rho}^{\tilde{c} \vee} \bar{\Psi}^{-1}, \bar{\rho}\right)$ by considering the linear extension $\widehat{R}$ of $R$ to $\mathcal{O}\left[G_{K}\right] \rightarrow$ $M_{2 n}(\mathcal{O})$. Put $T:=\operatorname{tr}(\widehat{R})$. Since $\widehat{R}$ is absolutely irreducible [2, Proposition 1.6.4] tells us that $\operatorname{ker} \widehat{R}=\operatorname{ker} T$. The morphism $\widehat{R}$ induces a morphism from $\mathcal{O}\left[G_{K}\right] / \operatorname{ker} \widehat{R}$ and so, applying [30, Theorem 1.1] again, we see that $c_{G}$ lies in the subspace

$$
\operatorname{Ext}_{\mathbf{F}\left[G_{K}\right] / \operatorname{ker} T}^{1}\left(\bar{\rho}^{\tilde{c} \vee} \bar{\Psi}^{-1}, \bar{\rho}\right) \subset \operatorname{Ext}_{\mathbf{F}\left[G_{K}\right]}^{1}\left(\bar{\rho}^{\tilde{c} \vee} \bar{\Psi}^{-1}, \bar{\rho}\right) .
$$

We conclude the proof using [2, Proposition 1.8.10(i)] which tells us that $\perp_{\tilde{c}}$ acts by multiplication by $\operatorname{sign}(\tilde{R}, \Psi, \tilde{c})$ on $\operatorname{Ext}_{\mathbf{F}\left[G_{K}\right] / \operatorname{ker} T}^{1}\left(\bar{\rho}^{\tilde{c} \vee} \bar{\Psi}^{-1}, \bar{\rho}\right)$. 
Under some mild additional assumptions one can check that the Theorem implies that this construction always produces elements in the Selmer group for the Asai motive that is critical in the sense of Deligne. For $\left.\Psi\right|_{G_{K}}=\epsilon^{-w}$ (e.g. the polarization character for the Galois representations associated to RACSDC representations of $\left.\mathrm{GL}_{w+1}\left(\mathbf{A}_{K}\right)\right)$ and $\operatorname{sign}(R, \Psi, \tilde{c})=1$ we have $\operatorname{As}^{-\Psi(\tilde{c}) \operatorname{sign}(R, \Psi, \tilde{c})}(\rho(i)) \otimes \Psi=$ $\operatorname{As}^{(-1)^{w+1}}(\rho)(-w+2 i)$. We can therefore deduce the following corollary to Theorem 4.1 and Proposition 3.3

Corollary 4.3. Let $p>2, K / K^{+} a C M$ field and $\rho: G_{K} \rightarrow \operatorname{GL}_{n}(E)$ be an residually irreducible continuous representation arising from a regular motive that is pure of some weight. Consider an absolutely irreducible representation

$$
R: G_{K} \rightarrow \mathrm{GL}_{2 n}(E)
$$

with $\operatorname{sign}(R, \Psi, \tilde{c})=1$ that is pure of weight $w$, unramified away from a finite set of places $\Sigma$ and short crystalline at $v \mid p$ and satisfies

$$
\left.R^{\vee} \cong R^{c} \otimes \Psi\right|_{G_{K}}
$$

with $\Psi: G_{K} \rightarrow E^{*}$ such that $\left.\Psi\right|_{G_{K}}=\epsilon^{-w}$. Assume that for some $i \in \mathbf{Z}$ such that $-w+2 i$ lies in the left-hand side of the critical strip of $\operatorname{As}(\rho)$ we have

$$
\bar{R}^{\mathrm{ss}}=\left.\bar{\rho}(i) \oplus \bar{\rho}^{c}(i)^{\vee} \otimes \bar{\Psi}^{-1}\right|_{G_{K}}
$$

and

$$
\bar{\rho}(i) \not \equiv \bar{\rho}^{c}(i)^{\vee} \bar{\Psi}^{-1} \bmod \varpi .
$$

If $\Sigma^{+}$is the set of places of $K^{+}$lying below $\Sigma$ then

$$
p \mid \# H_{\Sigma^{+}}^{1}\left(K^{+}, \operatorname{As}^{(-1)^{w+1}}(\rho)(-w+2 i) \otimes(E / \mathcal{O})\right)
$$

and $\mathrm{As}^{(-1)^{w+1}}(\rho)(-w+2 i)$ is critical.

Remark 4.4. By the Bloch-Kato Conjecture (Conjecture 3.1] the Selmer group in the corollary should be governed by the $L$-value $L\left(1+w-2 i, \pi, r^{(-1)^{w+1}}\right.$ ) (on the right-hand side of the critical strip).

Corollary 4.3 provides evidence for Conjecture 3.1 as we expect the existence of $R$ to be governed by the $p$-divisibility of the corresponding normalized Asai $L$-value. For $n=2$ and $i=0$ we described in [4, Secs. 6 and 7] a strategy to prove suitable congruences between the theta lift of the Bianchi modular form and stable Siegel cuspforms giving rise to $R$ as in the corollary.

\section{Fontaine-Mazur Conjecture}

By combining Fontaine-Mazur's conjecture ([14, Conjecture 1]) with Langlands automorphy conjectures one arrives at the following conjecture:

Conjecture 5.1. Let $R: G_{K} \rightarrow \mathrm{GL}_{m}\left(\overline{\mathbf{Q}}_{p}\right)$ be a continuous irreducible potentially semi-stable representation. Let $\Psi: G_{K^{+}} \rightarrow \overline{\mathbf{Q}}_{p}^{*}$ be a continuous character such that

$$
\left.R^{\vee} \cong R^{c} \otimes \Psi\right|_{G_{K}}
$$


Then there exists an algebraic essentially conjugate dual cuspidal automorphic representation $\pi$ of $\mathrm{GL}_{m} / K$ such that $\rho_{\pi} \cong R$.

Remark 5.2. Results toward this conjecture have been proven by Barnet-LambGee-Geraghty-Taylor [1] and Thorne [29]:

Theorem 4.5.1 of [1] proves potential automorphy for odd $R$ that are residually irreducible (and satisfying some further conditions, e.g. potential diagonalizability at $v \mid p)$.

Theorem 7.1 of 29] proves the conjecture for certain residually reducible $R$ with

$$
R^{\vee} \cong R^{c} \epsilon^{1-m}
$$

and $\bar{R}^{\mathrm{ss}} \cong \bar{\rho}_{1} \oplus \bar{\rho}_{2}$ with $\left.\bar{\rho}_{i}\right|_{G_{K}\left(\zeta_{p}\right)}$ adequate and $\bar{R}$ of Schur type, i.e. $\bar{\rho}_{1} \approx \bar{\rho}_{2}$ and $\epsilon^{1-m} \bar{\rho}_{1}^{c} \nRightarrow \bar{\rho}_{2}^{\vee}$ (so residual constituents do not get swapped by involution, different to our set-up). Apart from some other technical conditions 29] assumes $R$ ordinary at $v \mid p$ and that $\bar{R}^{\text {ss }}$ is automorphic, but not that $R$ is odd. The deformation theory techniques used to study residual representations of Schur type do not apply in our setting.

Similar to Calegari's result (see Theorem 1.1) the oddness of automorphic Galois representations (Theorem 2.6 and Remark 2.7) and Conjecture 5.1 implies the nonexistence of even geometric representations (except for certain exceptional cases like Tate twists of even representations with finite image). In the residually reducible case our result allows the following evidence toward Conjecture 5.1

Corollary 5.3. Let $(R, \Psi)$ be polarized as in Theorem 4.1 in particular such that

$$
\bar{R}^{\mathrm{ss}}=\left.\bar{\rho} \oplus \bar{\rho}^{c \vee} \otimes \Psi^{-1}\right|_{G_{K}} .
$$

If $H_{\Sigma^{+}}^{1}\left(K^{+}, \operatorname{As}^{\Psi(\tilde{c})}(\bar{\rho}) \otimes \bar{\Psi}\right)=0$ then $(R, \Psi)$ is totally odd.

Remark 5.4. Note that we can deduce total oddness by applying Theorem 4.1 for all complex conjugations $c_{v}$ for $v \mid \infty$, without a priori assuming the independence of $\Psi\left(c_{v}\right)$, since $H_{\Sigma^{+}}^{1}\left(K^{+}, \mathrm{As}^{\Psi\left(c_{v}\right)}(\rho) \otimes \Psi \otimes(E / \mathcal{O})\right)$ is independent of $v \mid \infty$. For this we note that the Galois representations $\operatorname{As}^{ \pm}(\rho)$ (defined as extensions of $\left.\rho \otimes \rho^{c_{v}}\right)$ are isomorphic for different $c_{v}$, and that $\operatorname{As}^{\Psi\left(c_{v}\right)}(\rho) \otimes \Psi$ is independent of $\Psi\left(c_{v}\right)$.

(2) Corollary 5.3 gives an alternative proof of Proposition 1.2 Let $\sigma: G_{\mathbf{Q}} \rightarrow$ $\mathrm{GL}_{2}\left(\overline{\mathbf{Q}}_{p}\right)$ be a short crystalline representation as in Proposition 1.2 and choose $K / \mathbf{Q}$ imaginary quadratic such that $R:=\left.\sigma\right|_{G_{K}}$ is irreducible (i.e. such that $\left.\sigma \neq \sigma \otimes \chi_{K / \mathbf{Q}}\right)$. We now take $K^{+}=\mathbf{Q}, n=1, \rho$ the trivial character $\mathbf{1}_{G_{K}}$ : $G_{K} \rightarrow \mathbf{Z}_{p}^{*}$, and $\Psi=\epsilon^{-m}$ in Corollary 5.3.

Note that $\mathrm{As}^{+}\left(\mathbf{1}_{G_{K}}\right)=\mathbf{1}_{G_{\mathbf{Q}}}$ and $\operatorname{As}^{\Psi(\tilde{c})}(\rho) \otimes \Psi=\chi_{K / \mathbf{Q}}^{m} \epsilon^{-m}$. Assume that $m$ is even (if $m$ is odd then there is nothing to show). Vandiver's conjecture then tells us that $p \nmid \operatorname{Cl}\left(\mathbf{Q}\left(\mu_{p}\right)\right)\left(\bar{\epsilon}^{-m}\right)$. Since $\mathbf{Q}\left(\mu_{p}\right)$ is a ramified extension of 
$\mathbf{Q}\left(\mu_{p}^{\otimes m}\right)$ we deduce that $p \nmid \mathrm{Cl}\left(\mathbf{Q}\left(\mu_{p}^{\otimes m}\right)\right)\left(\bar{\epsilon}^{-m}\right)$ also. The latter is isomorphic to $H_{f}^{1}\left(\mathbf{Q}, \mathbf{Q}_{p} / \mathbf{Z}_{p}\left(\bar{\epsilon}^{-m}\right)\right)$ (see e.g. [27] Proposition 1.6.2]). For $\Sigma^{+}=\{p\}$ we therefore have

$$
H_{f}^{1}\left(\mathbf{Q}, \mathbf{Q}_{p} / \mathbf{Z}_{p}\left(\bar{\epsilon}^{-m}\right)\right)[p]=H_{\Sigma^{+}}^{1}\left(\mathbf{Q}, \operatorname{As}^{\Psi(\tilde{c})}(\bar{\rho}) \otimes \bar{\Psi}\right)=0,
$$

so Corollary 5.3 implies that $R$ is odd with respect to $\Psi=\epsilon^{-m}$. As explained in Example 2.5 this would require $\Psi(\tilde{c})=-1$, a contradiction.

(3) If $(R, \Psi)$ satisfy further the assumptions of Corollary 4.3 (but without assuming that $(R, \Psi)$ is odd) then the Asai representation $\operatorname{As}^{\Psi(\tilde{c})}(\rho) \otimes \Psi$ in Corollary 5.3 is not critical. As explained in (2), in the particular case of $\epsilon^{-m}$ for even $m$ Vandiver's conjecture predicts that the non-divisibility assumption in Corollary 5.3 is satisfied.

(4) It is clear that some restriction on the tame levels included in $\Sigma^{+}$is needed so that the assumption $H_{\Sigma^{+}}^{1}\left(K^{+}, \mathrm{As}^{\Psi(\tilde{c})}(\bar{\rho}) \otimes \bar{\Psi}\right)=0$ can be satisfied. This corresponds to restrictions on the ramification of $R$ in Corollary [5.3, but as Remark 1.3 shows $\Sigma^{+} \not \supset\{p\}$ is possible.

\section{Acknowledgments}

The author would like to thank Gaëtan Chenevier, Neil Dummigan, Kris Klosin, Benoit Stroh, Jack Thorne and Jacques Tilouine for helpful conversations related to the topics of this paper, and the anonymous referee for several useful comments and corrections. The author's research was supported by the EPSRC First Grant $\mathrm{EP} / \mathrm{K} 01174 \mathrm{X} / 1$. The author would also like to thank ESI, Vienna, for their hospitality during the "Arithmetic Geometry and Automorphic Representations" workshop in April/May 2015, where part of this research was carried out.

\section{References}

[1] T. Barnet-Lamb, T. Gee, D. Geraghty and R. Taylor, Potential automorphy and change of weight, Ann. of Math. (2) 179(2) (2014) 501-609.

[2] J. Bellaïche and G. Chenevier, $p$-adic families of Galois representations and higher rank Selmer groups, Astérisque 324 (2009) xii+314.

[3] - The sign of Galois representations attached to automorphic forms for unitary groups, Compos. Math. 147(5) (2011) 1337-1352.

[4] T. Berger, On the Bloch-Kato conjecture for the Asai $L$-function, preprint (2015); http://arxiv.org/abs/1507.00684.

[5] T. Berger and K. Klosin, On deformation rings of residually reducible Galois representations and $R=T$ theorems, Math. Ann. 355(2) (2013) 481-518.

[6] S. Bloch and K. Kato, $L$-functions and Tamagawa numbers of motives, in The Grothendieck Festschrift, Vol. I, Progress in Mathematics, Vol. 86 (Birkhäuser Boston, Boston, MA, 1990), pp. 333-400.

[7] K. Buzzard and T. Gee, The conjectural connections between automorphic representations and Galois representations, in Automorphic Forms and Galois 
Representations. Vol. 1, London Mathematical Society Lecture Note Series, Vol. 414 (Cambridge University Press, Cambridge, 2014), pp. 135-187.

[8] F. Calegari, Even Galois representations (Lecture notes Poincare Institute), preprint (2011); http://www.math.northwestern.edu/ ${ }^{\sim}$ fcale/papers/FontaineTalkAdjusted.pdf.

[9] F. Calegari, Even Galois representations and the Fontaine-Mazur conjecture. II, J. Amer. Math. Soc. 25(2) (2012) 533-554.

[10] L. Clozel, Motifs et formes automorphes: applications du principe de fonctorialité, in Automorphic Forms, Shimura Varieties, and L-Functions, Vol. I, Perspectives in Mathematics, Vol. 10 (Academic Press, Boston, MA, 1990), pp. 77-159.

[11] L. Clozel, M. Harris and R. Taylor, Automorphy for some $l$-adic lifts of automorphic mod $l$ Galois representations, Publ. Math. Inst. Hautes Études Sci. 108 (2008) 1-181, with Appendix A, summarizing unpublished work of Russ Mann, and Appendix B by Marie-France Vignéras.

[12] F. Diamond, M. Flach and L. Guo, The Tamagawa number conjecture of adjoint motives of modular forms, Ann. Sci. École Norm. Sup. (4) 37 (2004) 663-727.

[13] N. Dummigan, Eisenstein congruences for unitary groups, preprint (2015); http://neil-dummigan.staff.shef.ac.uk/unitarycong4.pdf.

[14] J.-M. Fontaine and B. Mazur, Geometric Galois representations, in Elliptic Curves, Modular Forms, and Fermat's Last Theorem (International Press, Cambridge, MA, 1995), pp. 41-78.

[15] W. Goldring and J.-S. Koskivirta, Strata Hasse invariants, Hecke algebras and Galois representations, preprint (2015); http://arxiv.org/abs/1507.05032.

[16] N. Grbac and F. Shahidi, Endoscopic transfer for unitary groups and holomorphy of Asai L-functions, Pacific J. Math. 276(1) (2015) 185-211.

[17] M. Harris, L-functions and periods of adjoint motives, Algebra Number Theory $\mathbf{7}(1)$ (2013) 117-155.

[18] M. Harris, K.-W. Lan, R. Taylor and J. Thorne, On the rigid cohomology of certain Shimura varieties, Res. Math. Sci. 3(37) (2016) 1-308.

[19] C. Khare, Notes on Ribet's converse to Herbrand, in Cyclotomic Fields and Related Topics (Bhaskaracharya Pratishthana, Pune, 2000), pp. 273-284.

[20] M. Krishnamurthy, The Asai transfer to $\mathrm{GL}_{4}$ via the Langlands-Shahidi method, Int. Math. Res. Not. 2003(41) (2003) 2221-2254.

[21] Determination of cusp forms on $G L(2)$ by coefficients restricted to quadratic subfields (with an appendix by Dipendra Prasad and Dinakar Ramakrishnan), J. Number Theory 132(6) (2012) 1359-1384.

[22] V. Lafforgue, Chtoucas pour les groupes réductifs et paramétrisation de Langlands globale, preprint (2017); http://arxiv.org/pdf/1209.5352v8.pdf.

[23] V. Pilloni and B. Stroh, Cohomologie cohérente et représentations Galoisiennes, Ann. Math. Qué. 40(1) (2016) 167-202.

[24] D. Ramakrishnan, Modularity of solvable Artin representations of GO(4)-type, Int. Math. Res. Not. 2002(1) (2002) 1-54.

[25] Algebraic cycles on Hilbert modular fourfolds and poles of $L$-functions, in Algebraic Groups and Arithmetic (Tata Institute of Fundamental Research, Mumbai, 2004), pp. 221-274.

[26] K. A. Ribet, A modular construction of unramified p-extensions of $\mathbf{Q}\left(\mu_{p}\right)$, Invent. Math. 34(3) (1976) 151-162.

[27] K. Rubin, Euler Systems, Annals of Mathematics Studies, Vol. 147 (Princeton University Press, Princeton, NJ, 2000). 
[28] P. Scholze, On torsion in the cohomology of locally symmetric varieties, Ann. of Math. (2) $\mathbf{1 8 2 ( 3 )}$ (2015) 945-1066.

[29] J. A. Thorne, Automorphy lifting for residually reducible $l$-adic Galois representations, J. Amer. Math. Soc. 28(3) (2015) 785-870.

[30] E. Urban, Selmer groups and the Eisenstein-Klingen ideal, Duke Math. J. 106(3) (2001) 485-525. 